\title{
Application of PET/CT to adjuvant chemotherapy for early lung adenocarcinoma
}

\author{
Shinsuke Sasada, Yoshihiro Miyata, Takahiro Mimae, Yasuhiro Tsutani, Takeshi Mimura, Morihito Okada* \\ From World Society of Cardiothoracic Surgeons 25th Anniversary Congress, Edinburgh \\ Edinburgh, UK. 19-22 September 2015
}

\section{Background/Introduction}

The role of adjuvant chemotherapy for stage I lung cancer is unknown. Some Japanese trials demonstrated that tegaful-uracil chemotherapy improved the prognosis of stage I lung cancer over $2 \mathrm{~cm}$.

\section{Aims/Objectives}

The purpose of this study is to determine the significance of the maximum standardized uptake value (SUV$\max$ ) on F-18-fluorodeoxyglucose positron emission tomography/computed tomography (FDG-PET/CT) images to postoperative adjuvant chemotherapy for early lung adenocarcinoma.

\section{Method}

We reviewed 174 consecutive patients with completely resected pathological T1b-2aN0M0 lung adenocarcinoma between January 2006 and March 2011, and assessed recurrence-free interval and overall survival based on SUVmax values derived from preoperative FDG-PET/CT images. All patients were assessed by FDG-PET/CT before surgery

\section{Results}

Ninety patients received adjuvant chemotherapy and 84 did not. Patients given adjuvant chemotherapy were older, but had the lower $\mathrm{T}$ status tumor than patients who were not (both, $\mathrm{p}<0.001$ ). Adjuvant chemotherapy conferred benefits upon recurrence-free interval and overall survival compared with observation $(\mathrm{p}=0.007$ and $\mathrm{p}=0.004$, respectively). Multivariate Cox proportional hazard analyses revealed SUVmax as an independent prognostic factor for recurrence-free interval (hazard ratio 8.03, $\mathrm{p}<0.001$ ). Recurrence-free interval and overall survival were significantly longer for patients who received adjuvant

Department of Surgical Oncology, Hiroshima University, Hiroshima, 734-8551, Japan 